\title{
Cost effectiveness of ramipril treatment for cardiovascular risk reduction
}

\author{
I S Malik, V K Bhatia, J S Kooner
}

\begin{abstract}
Objective-To assess the cost effectiveness of ramipril treatment in patients at low, medium, and high risk of cardiovascular death.

Design-Population based cost effectiveness analysis from the perspective of the health care provider in the UK. Effectiveness was modelled using data from the HOPE (heart outcome prevention evaluation) trial. The life table method was used to predict mortality in a medium risk cohort, as in the HOPE trial (2.44\% annual mortality), and in low and high risk groups ( $1 \%$ and $4.5 \%$ annual mortality, respectively).

Setting-UK population using 1998 government actuary department data.

Main outcome measure-Cost per life year gained at five years and lifetime treatment with ramipril.

Results-Cost effectiveness was $£ 36600, £ 13$ 600, and $£ 4000$ per life year gained at five years and $£ 5300, £ 1900$, and $£ 100$ per life year gained at 20 years (lifetime treatment) in low, medium, and high risk groups, respectively. Cost effectiveness at 20 years remained well below that of haemodialysis ( $£ 25000$ per life year gained) over a range of potential drug costs and savings. Treatment of the HOPE population would cost the UK National Health Service (NHS) an additional $£ 360$ million but would prevent 12000 deaths per annum.

Conclusions-Ramipril is cost effective treatment for cardiovascular risk reduction in patients at medium, high, and low pretreatment risk, with a cost effectiveness comparable with the use of statins. Implementation of ramipril treatment in a medium risk population would result in a major reduction in cardiovascular deaths but would increase annual NHS spending by $£ 360$ million. (Heart 2001;85:539-543)
\end{abstract}

Keywords: angiotensin converting enzyme inhibitor; cardiovascular risk; cost effectiveness; ramipril

Coronary heart disease affects two million people and accounts for over 110000 deaths in the UK each year. ${ }^{1}$ Aspirin, ${ }^{2}$ hydroxymethyl glutaryl coenzyme A (HMG-CoA) reductase inhibitors (statins), ${ }^{3-5}$ and $\beta$ blockers ${ }^{6}$ reduce cardiovascular event rates in those at risk and are cost effective ${ }^{7-9}$ compared with recognised standards such as haemodialysis. ${ }^{10}$

The landmark HOPE (heart outcome prevention evaluation) showed that at five years' follow up ramipril treatment reduced cardiovascular events by $22 \%(\mathrm{p}<0.001)$ and total mortality by $16 \%(p=0.005)$ compared with placebo in patients with proven atherosclerotic disease (coronary artery disease, cerebrovascular disease, or peripheral vascular disease) or diabetes mellitus plus one additional vascular risk factor. ${ }^{11}$ The rates of revascularisation, angina, and heart failure were reduced in all groups and, in addition, in diabetic patients there was a reduction in the incidence of overt nephropathy. ${ }^{12}$ Implementation of the HOPE trial into clinical practice would make approximately three million people in the UK eligible for treatment with ramipril. ${ }^{13} 14$

The present study was designed to assess the cost effectiveness of ramipril treatment in the UK population in patients with low, medium, and high pretreatment cardiovascular risk on the basis of data from the HOPE trial.

\section{Methods}

CALCULATION OF COST EFFECTIVENESS

Cost effectiveness was assessed as the net cost in pounds per life year gained. The net cost was the cost of treatment minus the savings from reductions in treatment. The primary analysis was of net cost effectiveness with five year, 10 year, 15 year, and 20 year (lifetime) ramipril treatment assuming continued benefit from treatment. The effect of discounting estimates of life years gained, costs, and savings at $6 \%$ per annum was assessed ${ }^{15}{ }^{16}$ since benefits in future years may be valued less. Given the HOPE data, benefit is unlikely to decrease on continued treatment, but we modelled for the hypothesis that there would be no added benefit beyond five years' treatment. We did not use quality adjusted life years since no quality of life data were available from the HOPE trial.

ASSUMPTIONS IN THE ANALYSIS

Gain in life years using the life table method

The annual probability of dying at any age was calculated from age specific mortality rates for men in the UK population provided by the government actuary department. The average age of patients in the HOPE trial was 66 years. These patients had a mortality rate 1.31 times that of men aged 65-69 years in the UK general population, a ratio that was assumed to remain constant for life. The probability of dying in any given year in the cohort treated with ramipril was calculated by multiplying the annual probability in the placebo cohort by the relative risk of all cause mortality observed for treated patients in the HOPE trial, which was 0.84. Again this was assumed to remain constant for life. 
The life table method was used to calculate gain in life years. A cohort of patients aged 66 years, as in the HOPE trial, was taken and the mortality experienced on ramipril was compared with that on placebo treatment. Deaths in a given year occur at varying times. It was assumed that they occurred halfway through the year, on average, so that each death contributed half a year towards the total number of life years lived. The number of life years gained at the end of a year was the number of patients alive at the end of the year plus half a year for each death during that year. This process was repeated for the duration of the model. The net gain in life years was the total number of life years for the ramipril group minus the total for the placebo group. ${ }^{8}$

\section{Cost of treatment}

We assessed costs from the perspective of the health care provider. The majority of patients in the HOPE trial took ramipril $10 \mathrm{mg} /$ day. We used the price quoted in the British National Formulary $^{17}$ to calculate a cost of $£ 170$ per patient year. Drug cost was, therefore, the number of treatment years multiplied by the yearly cost of treatment. An additional physician consultation, pharmacy handling costs, and the need for one extra serum electrolytes measurement may raise total costs but the effect is small compared with drug price. ${ }^{18}$ To account for possible regional price differences, we assessed cost effectiveness at treatment prices varying between $50 \%$ and $200 \%$ of the British National Formulary stated cost of ramipril.

\section{Savings}

We assessed savings gained from reductions in myocardial infarction (from $12.0 \%$ to $9.8 \%$, $\mathrm{p}=0.0005)$, revascularisation $(18.4 \%$ to $16.0 \%, \mathrm{p}=0.0013)$, stroke $(4.8 \%$ to $3.3 \%$, $\mathrm{p}=0.0002)$, and angina as reported in the HOPE trial. Costs of these health care interventions were obtained from the literature (table 1). ${ }^{8}$ We did not include possible savings from reduction in incidence of diabetes mellitus or in overt nephropathy in patients with established diabetes. Actual charges made by an institution may differ from the unit cost of procedures. Based on current UK practice, revascularisation was assumed to be $50 \%$ percutaneous transluminal coronary angioplasty and $50 \%$ coronary artery bypass graft surgery, although the use of revascularisation procedures and the ratio of coronary angioplasty to coronary bypass surgery show regional variations. ${ }^{19}$ Estimates of cost savings were varied from $50-200 \%$ of initial values to allow for these differences.

PRETREATMENT LEVEL OF RISK

Patients in the HOPE trial were at moderate risk of cardiovascular events (placebo group annual mortality rate of $2.44 \%$ per year), similar to that experienced by the patients in the Scandinavian simvastatin survival study $(4 S){ }^{3}$ Since cost effectiveness is altered by pretreatment level of risk, ${ }^{8}$ we additionally assessed the cost effectiveness of ramipril treatment in low and high risk population groups, assuming ramipril to be equally effective in all patients.

Low risk group

We modelled the cost effectiveness in a low risk population with annual mortality rate of $1 \%$ at age 66 , a primary prevention group similar to the WOSCOPS (west of Scotland coronary outcome prevention study) population. ${ }^{7}$ This cohort on placebo had a mortality rate 0.54 times that of the age matched UK population since the patients enrolled in primary prevention trials are often more healthy than the general population.

\section{High risk group}

We modelled cost effectiveness in a high risk population with $4.5 \%$ annual mortality, equivalent to those who survive long term after thrombolysis for myocardial infarction. ${ }^{20}$ This cohort had a mortality rate on placebo 2.4 times that of the age matched UK population. Within this high risk population, we also modelled for a very high risk group, with $7 \%$ annual mortality, similar to patients with left ventricular dysfunction post-myocardial infarction in the AIRE (acute infarction ramipril efficacy) trial. $^{21}$

COST OF POPULATION TREATMENT

The prevalence of ischaemic heart disease, stroke, peripheral vascular disease, and diabetes was estimated using data from the health survey for England (1998) and the Scottish health survey (1995). ${ }^{22}$ For example, it has been estimated that $8.5 \%$ of men and $6.2 \%$ of women over 35 years old in England and Wales have had a myocardial infarction or stroke. ${ }^{13}$ These figures were used to calculate the cost of implementing ramipril treatment on a population basis. Data on mortality reduction from the HOPE trial were used to calculate the number of lives potentially saved by use of ramipril treatment in each group.

\section{Results}

BASE CASE ANALYSIS

Ramipril treatment within the medium risk group (the HOPE population) had a five year undiscounted cost effectiveness of $£ 13600$ per life year gained. Cost effectiveness improved to $£ 1900$ per life year gained with lifetime treatment (fig 1). Discounting at $6 \%$ reduced cost effectiveness to $£ 14700$ at five years and

Table 1 Published estimates of costs for coronary heart disease events and therapeutic procedures in UK pounds. Costs have been increased at the rate of inflation and reduced in line with the shorter duration of hospital stay in recent years ${ }^{8}$

\begin{tabular}{ll}
\hline Procedure & Cost $(£)$ \\
\hline Myocardial infarction & 1900 \\
PTCA & 3500 \\
CABG & 5500 \\
Average revascularisation (50\% PTCA: 50\% CABG) & 4500 \\
$\begin{array}{ll}\text { Other coronary disease related admissions (unstable } \\
\text { angina/heart failure) }\end{array}$ & 1500 \\
\hline
\end{tabular}

CABG, coronary artery bypass graft; PTCA, percutaneous transluminal coronary angioplasty. 


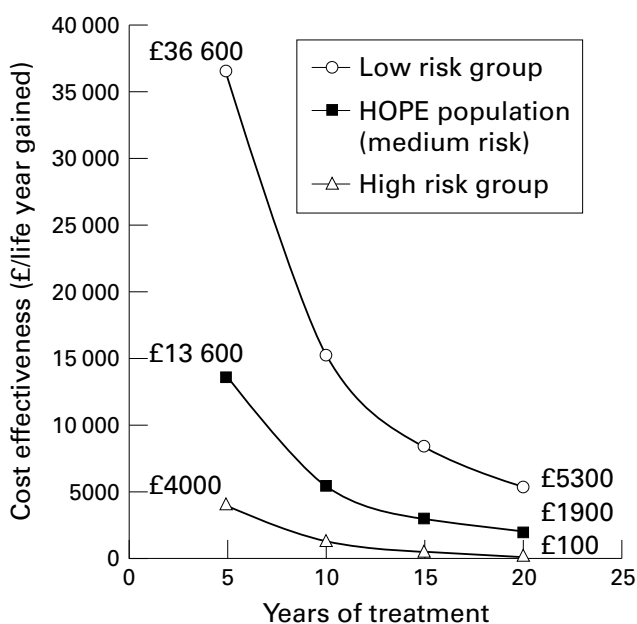

Figure 1 Cost effectiveness of ramipril treatment at 5, 10, 15 , and 20 years in patients with low (1.0\%), medium $(2.44 \%)$, and high (4.5\%) annual mortality rates. Haemodialysis has a cost effectiveness of $£ 25000$ per life year gained. For additional data see eHeart.

$£ 2800$ per life year gained for lifetime treatment. This is well below the threshold of $£ 25000$ per life year gained, which has been proposed to indicate cost effectiveness. ${ }^{10}$

EFFECT OF PRETREATMENT CARDIOVASCULAR RISK LEVEL

The low risk group had cost effectiveness of $£ 36600$ and $£ 5300$ per life year gained at five years and for lifetime treatment, respectively. The high risk group had a cost effectiveness of $£ 4000$ and $£ 100$ per /life year gained at five years and for lifetime treatment, respectively (fig 1). Treatment of the subgroup of patients at the highest risk ( $7 \%$ annual mortality) had a cost effectiveness of $£ 1300$ per life year gained at five years. Because of cumulative benefit, lifetime treatment has a cost effectiveness of minus $£ 900$ per life year over 20 years, a net cost saving.

SENSITIVITY TO COST OF DRUG AND POTENTIAL SAVINGS

Apart from pretreatment level of risk, the major determinant of the cost effectiveness of treatment was drug cost (fig 2). Varying the cost of

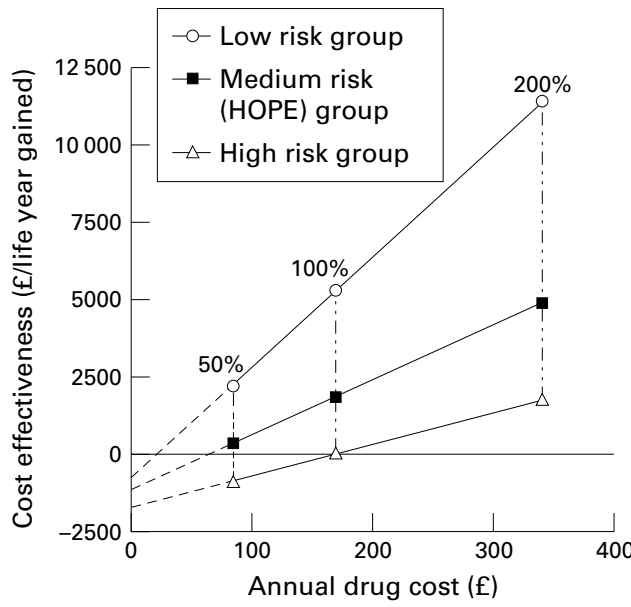

Figure 2 Cost effectiveness of lifelong (20 year) treatment with ramipril in low, medium, and high risk populations related drug cost at $50 \%, 100 \%$, and $200 \%$ of present prices. For additional data see eHeart.
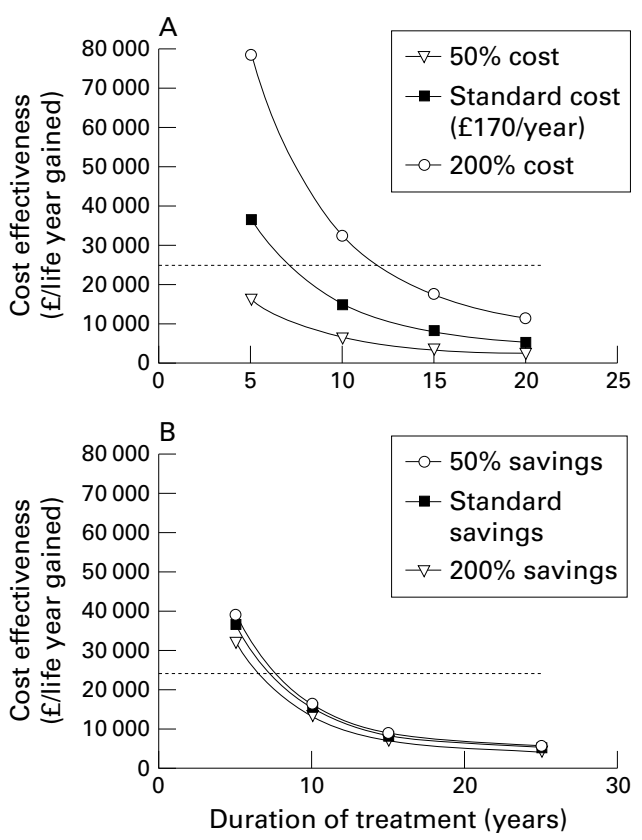

Figure 3 Cost effectiveness of ramipril treatment continued for $5,10,15$, and 20 years in a low risk population. Cost effectiveness of haemodialysis is $£ 25000$ per life year gained (shown as dotted line). (A) The effect of varying drug costs. (B) The effect of varying potential savings. For additional data see eHeart.

the drug in the range $50 \%$ to $200 \%$ of initial values altered lifetime cost effectiveness from $£ 400$ to $£ 4900$ per life year gained in the HOPE population. Changing the cost savings in the same range varied lifetime cost effectiveness from $£ 900$ to $£ 2500$ per life year gained.

For the low risk population, cost effectiveness at present drug costs was poor at five years. Adjusting drug cost between $50 \%$ and $200 \%$ of initial values altered lifetime cost effectiveness from $£ 2200$ to $£ 11400$ per life year gained (fig $3 \mathrm{~A}$ ) while varying potential savings over the same range resulted in cost effectiveness in the range $£ 4500$ to $£ 5700$ per life year gained (fig 3B).

\section{ALTERNATIVE MODELS}

Assuming that the HOPE population gained maximum benefit by five years and that there was no further gain from continuing medication, then cost effectiveness over 5-20 years remained stable at $£ 12000$ to $£ 13000$ per life year gained.

COST OF POPULATION TREATMENT

At least three million people in the UK may be eligible for, and may benefit from, treatment with ramipril following publication of the HOPE trial (table 2). Drug cost alone would increase National Health Service (NHS) spending by $£ 500$ million per year but, adjusting for possible reductions in health care use, net cost of treatment would be an additional $£ 360$ million per year. Ramipril treatment would save 12000 lives annually if the $1.8 \%$ absolute reduction in mortality seen over five years in the HOPE trial were realised. 
Table 2 Estimate of the number of patients eligible for ramipril treatment in the UK population. Data from the HOPE trial were used to quantify the number of lives gained annually on treatment

\begin{tabular}{lcllc}
\hline & $\begin{array}{l}\text { Eligible } \\
\text { population }\end{array}$ & $\begin{array}{l}\text { New prescriptions } \\
(\%)\end{array}$ & $\begin{array}{l}\text { Net cost (total cost) } \\
(£ \text { million) }\end{array}$ & $\begin{array}{l}\text { Lives gained } \\
\text { per year }\end{array}$ \\
\hline Total population & $\geqslant 3000000$ & 100 & $500(500)$ & 12000 \\
IHD $^{31}$ & 1400000 & $70^{30}$ & $166(240)$ & 5600 \\
Stroke $^{21}$ & 600000 & 95 & $100(102)$ & 2400 \\
Diabetes $^{21}$ & 1700000 & 50 & $144(288)$ & 6800 \\
PVD $^{21}$ & 1000000 & 99 & $180(180)$ & 4000 \\
\hline
\end{tabular}

IHD, ischaemic heart disease; PVD, peripheral vascular disease.

\section{Discussion}

COST EFFECTIVENESS OF RAMIPRIL IN THE HOPE POPULATION

The present study shows that ramipril is a cost effective treatment for patients with proven vascular disease or diabetes mellitus plus an additional risk factor. Treatment with ramipril to reduce cardiovascular risk would cost $£ 13600$ per life year gained over five years and $£ 1900$ per life year gained for 20 years of treatment. These results suggest that ramipril treatment has a cost effectiveness similar to that of other medical treatments (fig 4) and substantially below the standard of $£ 25000$ per life year gained set by haemodialysis. ${ }^{10}$ Direct comparison of the cost effectiveness of ramipril with that of other treatments has limitations because the model used varies between studies. $^{23}$ Nevertheless, ramipril treatment compared favourably with statin treatment, which had a lifetime cost effectiveness of $£ 8200$ per life year gained in a study of a medium risk population using a model similar to our own. ${ }^{8}$ Reduction in drug cost would improve cost effectiveness further and may occur with prescription of generic drugs or use of less expensive angiotensin converting enzyme (ACE) inhibitors if they are shown to have effects similar to those of ramipril. ${ }^{24}$ In fact, life years might be gained at zero cost if the price of ramipril falls below $£ 62$ per year (fig 2).

EFFECT OF PRETREATMENT CARDIOVASCULAR RISK LEVEL

Previous studies have shown that ramipril is a cost effective treatment in patients with left

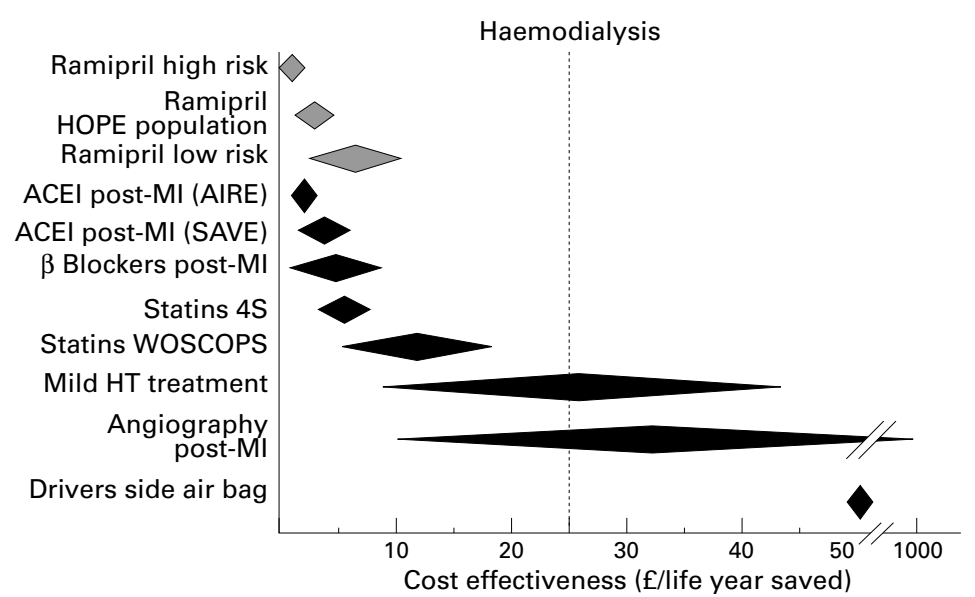

Figure 4 Comparison of cost effectiveness of ramipril treatment with other cardiovascular and non-cardiovascular interventions. Estimates from previous studies are in 1994-1995 UK pounds. ${ }^{79} 232435$ ACEI, angiotensin converting enzyme inhibitor; HT, hypertension; MI, myocardial infarction. See text for explanation of trial acronyms. ventricular dysfunction post-myocardial infarction. ${ }^{2526}$ Our results in high risk subgroups are consistent with these findings. In addition, we have shown that in a low risk population, lifelong ramipril treatment to reduce cardiovascular events is cost effective over a range of estimates for drug costs and savings.

\section{MODEL ASSUMPTIONS}

The HOPE study population has been followed up for a mean of five years to date. A possible limitation of the present analysis is the projection of cost effectiveness to 10,15 , and 20 years' treatment based on the assumption that the benefits from ramipril would persist beyond five years. ${ }^{27}$ The reduction in mortality seen in the HOPE trial, although a secondary end point, is in keeping with previous data suggesting that there is cardiovascular event reduction with $\mathrm{ACE}$ inhibitors. ${ }^{28-30}$ The relative risk reduction in mortality with ramipril treatment was assumed to be identical for all population groups treated. For example, our very high risk subgroup (with a mortality rate similar to that of patients recruited into the AIRE trial $^{31}$ ) was assigned a $16 \%$ reduction in total mortality on treatment as in the HOPE trial, rather than the $27 \%$ reduction seen in the AIRE trial. Cost effectiveness for the highest risk group may, therefore, have been underestimated. The cost of treatment may also have been overestimated in our analysis because no account was taken of the $30 \%$ of patients in the HOPE trial who were non-compliant or withdrew from treatment. The micro-HOPE study showed a $24 \%$ reduction in the incidence of overt nephropathy in diabetic patients on ramipril treatment versus placebo. ${ }^{12}$ No account has been taken of reduction in complications related to diabetes mellitus. Benefits may have been underestimated and cost overestimated in our analysis. Our data, therefore, present a conservative estimate of actual cost effectiveness.

Our results are valid for the UK but they may not be transferable to other countries by simple currency conversion because approaches to health care (such as assessment of need for interventions and the costs of such interventions) vary considerably.

IMPLEMENTING COST EFFECTIVE TREATMENT Up to three million of the UK population may be eligible for, and benefit from, ramipril treatment. ${ }^{14}$ Of this medium risk group, fewer than $30 \%$ are taking an ACE inhibitor. ${ }^{32}$ The proportion is substantially lower for patients with peripheral vascular disease and stroke. Vascular diseases already cost the NHS f3.8 billion per year. ${ }^{33} \mathrm{~A}$ further $£ 360$ million would be required to treat these patients. However, treatment would save an estimated 12000 lives per year (table 2). Joint guidelines by four British societies and the British Hypertension Society guidelines recommend statin treatment when coronary artery disease risk is greater than $30 \%$ over 10 years. $^{34}$ These guidelines were based on data suggesting that statin treatment is both clinically effective and cost effective. The HOPE trial confirmed that ramipril 
reduced cardiovascular risk. We have shown that treatment has a cost effectiveness similar to that of statin treatment.

In conclusion, ramipril is cost effective treatment for patients with proven vascular disease or diabetes mellitus plus an additional risk factor and has a cost effectiveness similar to the use of statins for cardiovascular risk reduction. Even for patients at lower risk, cost effectiveness for lifelong treatment is well below $£ 25000$ per life year gained, a standard below which treatment is considered acceptable. Implementation of the findings of the HOPE trial may save 12000 lives per year in the UK but would increase by $10 \%$ the NHS spending on vascular disease.

IM and VB are supported by Junior Research Fellowships from the British Heart Foundation.

1 Anon. National service framework for coronary heart disease. Anon. National service framework for coronary heart disease.
London: Department of Health, Government stationery London: Depar
office, 2000

2 Collaborative overview of randomised trials of antiplatelet therapy-I: prevention of death, myocardial infarction, and stroke by prolonged antiplatelet therapy in various categories of patients. Antiplatelet trialists' collaboration. $B M \mathcal{F}$ 1994;308:81-106

3 Randomised trial of cholesterol lowering in 4444 patients with coronary heart disease: the Scandinavian simvastatin survival study (4S). Lancet 1994;344:1383-9.

4 Sacks FM. The effect of pravastatin on coronary events after myocardial infarction in patients with average cholesterol levels. N Engl f Med 1997;335:1001-9.

5 Shepherd J, Cobbe SM, Ford I, et al. Prevention of coronary heart disease with pravastatin in men with hypercholesterolemia. West of Scotland coronary prevention study group. $\mathrm{N} \mathrm{Engl} \mathrm{f} \mathrm{Med} \mathrm{1995;333:1301-7.}$

6 Freemantle N, Cleland J, Young P, et al. Beta blockade after myocardial infarction: systematic review and meta myocardial infarction: systematic review

7 Caro J, Klittich W, McGuire A, et al. The west of Scotland coronary prevention study: economic benefit analysis of primary prevention with pravastatin. BMf 1997;315:1557-

8 Pickin DM, McCabe CJ, Ramsay LE, et al. Cost effectiveness of HMG-CoA reductase inhibitor (statin) treatment related to the risk of coronary heart disease and cost of drug treatment. Heart 1999;82:325-32.

9 Jonsson B, Johannesson M, Kjekshus J, et al. Costeffectiveness of cholesterol lowering. Results from the Scandinavian simvastatin survival study (4S). Eur Heart $\mathcal{F}$ 1996;17:1001-7.

10 Mark DB. Medical economics in cardiovascular medicine. In: Topol EJ, ed. Textbook of cardiovascular medicine. New York: Lippincott-Raven, 1997:1033-62.

11 Yusuf S, Sleight P, Pogue J, et al. Effects of an angiotensinconverting-enzyme inhibitor, ramipril, on cardiovascular events in high-risk patients. The Heart outcomes prevenevents in high-risk patients. The Heart outcomes preven-
tion evaluation study investigators $N$ Engl f Med 2000;342: tion evaluat $145-53$.

website

extra

Supplemental data for figs 1-3 appear on the Heart website

www.heartjnl.com

12 Heart Outcomes Prevention Evaluation Study Investigators. Effects of ramipril on cardiovascular and microvascular outcomes in people with diabetes mellitus: results of the HOPE study and MICRO-HOPE substudy. Lancet 2000; 355:253-9.

13 National Centre for Social Research. Health Survey for England: Cardiovascular disease '98. London: Department of Health, 2000
14 Ul-Haq I, Ramsay LE, Pickin DM. Lipid-lowering for prevention of coronary heart disease: what policy now? Clin Sci (Colch) 1996;91:399-413.

15 Anon. Appraisal and evaluation in central government. London: Government Stationery Office, 1997.

16 Spackman M. Discount rates and rates of return in the public sector: economic issues [Working paper 113]. London: Government Economic Service, 1991

17 Anon. British National Formulary. London: BMJ Books/ Pharmaceutical Press, 2000.

18 Federal Register. Washington: US Government Printing Office, 1994.

19 Audit Commission. Dear to our hearts? Commissioning services for the treatment and prevention of coronary heart disease. London: HMSO, 1995.

20 Baigent C, Collins R, Appleby P. ISIS-2: 10 year survival among patients with suspected acute myocardial infarction in randomised comparison of intravenous streptokinase, oral aspirin, both or neither. BMF 1998;316:1337-43.

21 Hall AS, Murray GD, Ball SG. Follow-up study of patients randomly allocated ramipril or placebo for heart failure after acute myocardial infarction: AIRE extension (AIREX) study. Acute infarction ramipril efficacy. Lancet 1997;349:1493-7

22 Anon. Scottish health survey - 1995. London: HMSO, 1997.

3 Mason J, Drummond M, Torrance G. Some guidelines on the use of cost effectiveness league tables. BMF 1993;306: $570-2$.

24 Fox KM, Henderson JR, Bertrand ME, et al. The European trial on reduction of cardiac events with perindopril in stable coronary artery disease (EUROPA). Eur Heart $\mathcal{f}$ 1998;19(suppl J):J52-5.

25 Erhardt L, Ball S, Andersson F, et al. Cost effectiveness in the treatment of heart failure with ramipril. A Swedish substudy of the AIRE study. Acute infarction ramipril efficacy. Pharmacoeconomics 1997;12:256-66.

26 Schadlich PK, Huppertz E, Brecht JG. Cost-effectiveness analysis of ramipril in heart failure after myocardial infarction. Economic evaluation of the acute infarction ramipril efficacy (AIRE) study for Germany from the perspective of statutory health insurance. Pharmacoeconomics 1998;14: 653-69.

27 Swedberg K, Kjekshus J, Snapinn S. Long-term survival in severe heart failure in patients treated with enalapril. Ten year follow-up of CONSENSUS I. Eur Heart 7 1999;20: 136-9.

28 GISSI III. Effect of the ACEI lisinopril on mortality in diabetic patients with acute myocardial infarction-GISSI III data. Circulation 1997;96:4239-45.

29 Rutherford JD, Pfeffer MA, Moye LA, et al. Effects of captopril on ischemic events after myocardial infarction. Results of the survival and ventricular enlargement trial. SAVE investigators. Circulation 1994;90:1731-8.

30 ISIS-4 Collaborative Group. Fourth International study of infarct survival: protocol for a large simple study of the effects of oral mononitrate, of oral captopril, and of intravenous magnesium. ISIS-4 collaborative group. Am $\mathcal{F}$ Cardiol 1991:68:87D-100D.

31 Effect of ramipril on mortality and morbidity of survivors of acute myocardial infarction with clinical evidence of heart failure. The acute infarction ramipril efficacy (AIRE) study investigators. Lancet 1993;342:821-8.

32 EUROASPIRE Study Group. A European Society of Cardiology survey of secondary prevention of coronary heart disease: principal results. EUROASPIRE study group. European Action on secondary prevention through intervention to reduce events. Eur Heart 7 1997; 18:1569-82.

33 Anon. National service framework: emerging findings report. London: HMSO, 1998.

34 Joint British recommendations on prevention of coronary heart disease in clinical practice. British Cardiac Society, British Hyperlipidaemia Association, British Hypertension Society, endorsed by the British Diabetic Association. Heart 1998;80 (suppl 2):S1-29.

35 Goldman L, Sia ST, Cook EF, et al. Costs and effectiveness of routine therapy with long-term beta-adrenergic antagonists after acute myocardial infarction. $N$ Engl f Med 1988; 319:152-7. 